\title{
Spin Resistivity in Magnetic Materials
}

\author{
H.T. DieP*, Y. MaGnin ${ }^{\dagger}$ And Danh-Tai Hoang \\ Laboratoire de Physique Théorique et Modélisation, Université de Cergy-Pontoise, CNRS, UMR 8089 \\ 2, Av. Adolphe Chauvin, 95302 Cergy-Pontoise Cedex, France
}

\begin{abstract}
We show in this paper recent results on the spin resistivity in magnetically ordered materials obtained by Monte Carlo simulations. We discuss its behavior as a function of temperature in various types of crystal: ferromagnetic, antiferromagnetic, and frustrated spin systems. In the model used for simulations, we take into account the interaction between itinerant spins and that between lattice spins and itinerant spins. We also include a chemical potential term, as well as an electric field. We study in particular the behavior of the spin resistivity at and near the magnetic phase transition where the effect of the magnetic ordering is strongest. In ferromagnetic crystals, the spin resistivity shows a sharp peak very similar to the magnetic susceptibility. This can be understood if one relates the spin resistivity to the spin-spin correlation as suggested in a number of theories. The dependence of the shape of the peak on physical parameters such as carrier concentration, magnetic field strength, relaxation time etc. is discussed. In antiferromagnets, the peak is not so pronounced and in some cases it is absent. Its direct relationship to the spin-spin correlation is not obvious. As for frustrated spin systems with strong first-order transition, the spin resistivity shows a discontinuity at the phase transition. To show the efficiency of the simulation method, we compare our results with recent experimental data performed on semiconducting MnTe of NiAs structure. We observe a very good agreement with experiments on the spin resistivity in the whole range of temperature.
\end{abstract}

PACS: $75.76 .+\mathrm{j}, 05.60 . \mathrm{Cd}$

\section{Introduction}

The study of the behavior of the resistivity is one of the fundamental tasks in materials science. This is because the transport properties occupy the first place in electronic devices and applications. The resistivity has been studied since the discovery of the electron a century ago by the simple Drude theory using the classical free particle model with collisions due to atoms in the crystal. The following relation is established between the conductivity $\sigma$ and the electronic parameters $e$ (charge) and $m$ (mass):

$$
\sigma=\frac{n e^{2} \tau}{m}
$$

where $\tau$ is the electron relaxation time, namely the average time between two successive collisions. In more sophisticated treatments of the resistivity where various interactions are taken into account, this relation is still valid provided two modifications: (i) the electron mass is replaced by its effective mass which includes various effects due to interactions with its environment, (ii) the relaxation time $\tau$ is not a constant but dependent on col-

\footnotetext{
* corresponding author; e-mail: diep@u-cergy.fr

$\dagger$ Also at Asia Pacific Center for Theoretical Physics, Hogil Kim Memorial Bldg. \#501, POSTECH San 31 Hyoja-dong, Nam-gu, Pohang Gyeongbuk 790-784, Korea.
}

lision mechanisms. The first modification is very important, the electron can have a "heavy" or "light" effective mass which modifies its mobility in crystals. The second modification has a strong impact on the temperature dependence of the resistivity: $\tau$ depends on some power of the electron energy, this power depends on the diffusion mechanisms such as collisions with charged impurities, neutral impurities, magnetic impurities, phonons, magnons, etc. As a consequence, the relaxation time averaged over energy, $\langle\tau\rangle$, depends differently on $T$ according to the nature of the collision source. The properties of the total resistivity stem thus from different kinds of diffusion processes. Each contribution has in general a different temperature dependence.

Let us summarize the most important contributions to the total resistivity $\rho_{\mathrm{t}}(T)$ at low temperature $(T)$ in the following expression:

$$
\rho_{\mathrm{t}}(T)=\rho_{0}+A_{1} T^{2}+A_{2} T^{5}+A_{3} \ln \frac{\mu}{T},
$$

where $A_{1}, A_{2}$ and $A_{3}$ are constants. The first term is $T$-independent, the second term proportional to $T^{2}$ represents the scattering of itinerant spins at low $T$ by lattice spin waves. Let us note that the resistivity caused by a Fermi liquid is also proportional to $T^{2}$. The $T^{5}$ term corresponds to low- $T$ resistivity in metals. This is due to the scattering of itinerant electrons by phonons. Let us note that at high $T$, metals show a linear- $T$ dependence. The $\ln$ term is the resistivity due to the quantum Kondo effect caused by a magnetic impurity at very low $T$. 
We are interested here in the spin resistivity $\rho$ of magnetic materials. This subject has been investigated intensively both experimentally and theoretically for more than five decades. The rapid development of the field is due mainly to many applications in particular in spintronics.

Experiments have been performed in many magnetic materials including metals, semiconductors and superconductors. One interesting aspect of magnetic materials is the existence of a magnetic phase transition from a magnetically ordered phase to the paramagnetic (disordered) state. Very recent experiments such as those performed on the following compounds show different forms of anomaly of the magnetic resistivity at the magnetic phase transition temperature: ferromagnetic $\mathrm{SrRuO}_{3}$ thin films [1], Ru-doped induced ferromagnetic $\mathrm{La}_{0.4} \mathrm{Ca}_{0.6} \mathrm{MnO}_{3}$ [2], antiferromagnetic $\epsilon$ - $\left(\mathrm{Mn}_{1-x} \mathrm{Fe}_{x}\right)_{3.25} \mathrm{Ge}$ [3], semiconducting $\operatorname{Pr}_{0.7} \mathrm{Ca}_{0.3} \mathrm{MnO}_{3}$ thin films [4], superconducting $\mathrm{BaFe}_{2} \mathrm{As}_{2}$ single crystals [5], and $\mathrm{La}_{1-x} \mathrm{Sr}_{x} \mathrm{MnO}_{3}$ [6]. Depending on the material, $\rho$ can show a sharp peak at the magnetic transition temperature $T_{\mathrm{C}}$ [7] or just only a change of its slope, or an inflexion point. The latter case gives rise to a peak of the differential resistivity $\mathrm{d} \rho / \mathrm{d} T$ $[8,9]$.

As for theories, the $T^{2}$ magnetic contribution in Eq. (2) has been obtained from the magnon scattering by Kasuya [10]. However, at high $T$ in particular in the region of the phase transition, much less has been known. de Gennes and Friedel [11] proposed this idea that the magnetic resistivity results from the spin-spin correlation so it should behave as the magnetic susceptibility, thus it should diverge at $T_{\mathrm{C}}$. Fisher and Langer [12], and Kataoka [13] have suggested that the range of spin-spin correlation changes the shape of $\rho$ near the phase transition. The resistivity due to magnetic impurities has been calculated by Zarand et al. [14] as a function of the Anderson localization length. This parameter expresses in fact a kind of the correlation sphere induced around each impurity. Their result shows that the resistivity peak depends on this parameter, in agreement with the spin-spin correlation idea.

The absence of Monte Carlo (MC) simulation in the literature on the spin transport has motivated our recent works: we have studied the spin current in ferromagnetic [15-17] and antiferromagnetic [18-21] materials by MC simulations. The behavior of $\rho$ as a function of $T$ has been shown to be in agreement with main experimental features and theoretical investigations mentioned above.

In this paper, we give a review of these works, outline the most important aspects and results. We consider in some details the case of MnTe where our simulation is in excellent agreement with experiments.

In Sect. 2, we show our basic model and describe our MC method. Results are shown and discussed in Sect. 3. The case of MnTe is considered in Sect. 3.3. Concluding remarks are given in Sect. 4 .

\section{Model and Method}

\subsection{Model}

The model used in our MC simulation is very general. The itinerant spins move in a crystal whose lattice sites are occupied by localized spins. The itinerant spins and the localized spins may be of Ising, $X Y$ or Heisenberg models. Their interaction is usually limited to nearest neighbors (NN) but this assumption is not necessary. It can be ferromagnetic or antiferromagnetic.

Let us note that the purpose of this paper is to study the effect of the magnetic transition on $\rho$. This transition occurs at a high temperature where it is known that the quantum nature of itinerant electron spins does not make significant additional effects with respect to the classical spin model. Therefore, to simplify the task, we consider here the classical spin model.

\subsubsection{Interactions}

We consider a crystal of a given lattice structure where each lattice site is occupied by a spin. The interaction between the lattice spins is given by the following Hamiltonian:

$$
\mathcal{H}_{l}=-\sum_{(i, j)} J_{i, j} \boldsymbol{S}_{i} \cdot \boldsymbol{S}_{j},
$$

where $\boldsymbol{S}_{i}$ is the spin localized at lattice site $i$ of the Ising, $X Y$ or Heisenberg model, $J_{i, j}$ — the exchange integral between the spin pair $\boldsymbol{S}_{i}$ and $\boldsymbol{S}_{j}$ which is not limited to the interaction between nearest-neighbors (NN). Hereafter, except otherwise stated, we take $J_{i, j}=J$ for NN spin pairs, for simplicity. $J>0(<0)$ denotes ferromagnetic (antiferromagnetic) interaction. The system size is $L_{x} \times L_{y} \times L_{z}$ where $L_{i}(i=x, y, z)$ is the number of lattice cells in the $i$ direction. Periodic boundary conditions $(\mathrm{PBC})$ are used in all directions.

We define the interaction between itinerant spins and localized lattice spins as follows:

$$
\mathcal{H}_{r}=-\sum_{i, j} I_{i, j} \boldsymbol{\sigma}_{i} \cdot \boldsymbol{S}_{j},
$$

where $\boldsymbol{\sigma}_{i}$ is the spin of the $i$-th itinerant electron and $I_{i, j}$ denotes the interaction which depends on the distance between electron $i$ and spin $\boldsymbol{S}_{j}$ at lattice site $j$. For simplicity, we suppose the following interaction expression:

$$
I_{i, j}=I_{0} \mathrm{e}^{-\alpha r_{i j}},
$$

where $r_{i j}=\left|\boldsymbol{r}_{i}-\boldsymbol{r}_{j}\right|, I_{0}$ and $\alpha$ are constants. We use a cut-off distance $D_{1}$ for the above interaction. In the same way, interaction between itinerant electrons is defined by

$$
\begin{aligned}
\mathcal{H}_{m} & =-\sum_{i, j} K_{i, j} \boldsymbol{\sigma}_{i} \cdot \boldsymbol{\sigma}_{j}, \\
K_{i, j} & =K_{0} \mathrm{e}^{-\beta r_{i j}},
\end{aligned}
$$

with $K_{i, j}$ being the interaction between electrons $i$ and $j$, limited in a sphere of radius $D_{2}$. The choice of the constants $K_{0}$ and $\beta$ will be discussed below.

Let us note that the choice of an exponential law does not affect the general feature of our results presented in 
this paper because the short cut-off distance used here limits the interaction to a small number of neighbors, typically to next nearest neighbors (NNN), so the choice of another law such as a power law, or even discrete interaction values, for such a small cut-off will not make a qualitative difference in the results.

Itinerant electrons move under an electric field applied along the $x$ axis. The PBC ensure that the electrons who leave the system at one end are to be reinserted at the other end. These boundary conditions are used in order to conserve the average density of itinerant electrons. One has

$$
\mathcal{H}_{\mathrm{E}}=-e \boldsymbol{\epsilon} \cdot \boldsymbol{\ell},
$$

where $e$ is the electronic charge, $\boldsymbol{\epsilon}-$ an applied electric field and $\ell-$ a displacement vector of an electron.

Since the interaction between itinerant electron spins is attractive, we need to add a kind of "chemical potential" in order to avoid a possible collapse of electrons into some points in the crystal and to ensure a homogeneous spatial distribution of electrons during the simulation. The chemical potential term is given by

$$
\mathcal{H}_{\mathrm{c}}=D\left[n(\boldsymbol{r})-n_{0}\right],
$$

where $n(\boldsymbol{r})$ is the concentration of itinerant spins in the sphere of $D_{2}$ radius, centered at $\boldsymbol{r}, n_{0}$ - the average concentration, and $D$ - a constant parameter.

\subsubsection{Choice of parameters and units}

As mentioned earlier, our model is very general. Several kinds of materials such as metals, semiconductors, insulating magnetic materials etc. can be studied with this model, provided an appropriate choice of the parameters. For example, non-magnetic metals correspond to $I_{i, j}=K_{i, j}=0$ (free conduction electrons). Magnetic semiconductors correspond to the choice of parameters $K_{0}$ and $I_{0}$ so as the energy of an itinerant electron due to the interaction $\mathcal{H}_{r}$ should be much lower than that due to $\mathcal{H}_{m}$, namely itinerant electrons are more or less bound to localized atoms. Let us note that $\mathcal{H}_{m}$ depends on the concentration of itinerant spins: for example the dilute case yields a small $\mathcal{H}_{m}$. We make simulations for typical values of parameters which correspond to semiconductors. The choice of the parameters has been made after numerous test runs. We describe the principal requirements which guide the choice: (i) we choose the interaction between lattice spins as unity, i.e. $|J|=1$, (ii) we choose interaction between an itinerant and its surrounding lattice spins so as its energy $E_{i}$ in the low $T$ region is the same order of magnitude with that between lattice spins. To simplify, we take $\alpha=1$. This case corresponds to a semiconductor, as mentioned earlier, (iii) interaction between itinerant spins is chosen so that this contribution to the itinerant spin energy is smaller than $E_{i}$ in order to highlight the effect of the lattice ordering on the spin current. To simplify, we take $\beta=1$, (iv) the choice of $D$ is made in such a way to avoid the formation of clusters of itinerant spins (agglomeration) due to their attractive interaction [Eq. (7)], (v) the electric field is chosen not so strong in order to avoid its dominant effect that would mask the effects of thermal fluctuations and of the magnetic ordering, (vi) the density of the itinerant spins is chosen in a way that the contribution of interactions between themselves is much weaker than $E_{i}$, as mentioned above in the case of semiconductors.

Within the above requirements, a variation of each parameter does not change qualitatively the results shown below. Only the variation of $D_{1}$ in some antiferromagnets does change the results (see Ref. [20]).

The energy is measured in the unit of $|J|$. The temperature is expressed in the unit of $|J| / k_{\mathrm{B}}$. The distance $\left(D_{1}\right.$ and $\left.D_{2}\right)$ is in the unit of the lattice constant $a$. Real units will be used in Sect. 3.3 for comparison with experiments.

\subsection{Simulation method}

Using the Metropolis algorithm, we first equilibrate the lattice at a given temperature $T$ without itinerant electrons. When equilibrium is reached, we randomly add $N_{0}$ polarized itinerant spins into the lattice. Each itinerant electron interacts with lattice spins in a sphere of radius $D_{1}$ centered at its position, and with other itinerant electrons in a sphere of radius $D_{2}$. We next equilibrate the itinerant spins using the following updating. We calculate the energy $E_{\text {old }}$ of an itinerant electron taking into account all interactions described above. Then we perform a trial move of length $\ell$ taken in an arbitrary direction with random modulus in the interval $\left[R_{1}, R_{2}\right]$ where $R_{1}=0$ and $R_{2}=a$ (NN distance), $a$ being the lattice constant. Let us note that the move is rejected if the electron falls in a sphere of radius $r_{0}$ centered at a lattice spin or at another itinerant electron. This excluded space emulates the Pauli exclusion. We calculate the new energy $E_{\text {new }}$ and use the Metropolis algorithm to accept or reject the electron displacement. We choose another itinerant electron and begin again this procedure. When all itinerant electrons are considered, we say that we have made a MC sweeping, or one MC step/spin. We have to repeat a large number of MC steps/spin to reach a stationary transport regime. We then perform the averaging to determine physical properties such as magnetic resistivity, electron velocity, energy etc. as functions of temperature. We define the dimensionless spin resistivity $\rho$ as

$$
\rho=\frac{1}{n_{\mathrm{e}}},
$$

where $n_{\mathrm{e}}$ is the number of itinerant electron spins crossing a unit slice perpendicular to the $x$ direction per unit of time. An example with real units is shown in Sect. 3.3.

In order to have sufficient statistical averages on microscopic states of both the lattice spins and the itinerant spins, we use what we call "multi-step averaging procedure": after averaging the resistivity over $N_{1}$ steps for "each" lattice spin configuration, we thermalize again the lattice with $N_{2}$ steps in order to take another disconnected lattice configuration. Then we take back the 
averaging of the resistivity for $N_{1}$ steps for the new lattice configuration. We repeat this cycle for $N_{3}$ times, usually several hundreds of thousands times. The total MC steps for averaging is about $4 \times 10^{5}$ steps per spin in our simulations. This procedure reduces strongly thermal fluctuations observed in our previous work [16].

Of course, the larger $N_{2}$ and $N_{3}$ are, the better the statistics become. The question is what is the correct value of $N_{1}$ for averaging with one lattice spin configuration at a given $T$ ? This question is important because this is related to the relaxation time $\tau_{\mathrm{L}}$ of the lattice spins compared to that of the itinerant spins, $\tau_{\mathrm{I}}$. The two extreme cases are (i) $\tau_{\mathrm{L}} \simeq \tau_{\mathrm{I}}$, one should take $N_{1}=1$, namely the lattice spin configuration should change with each move of itinerant spins, (ii) $\tau_{\mathrm{L}} \gg \tau_{\mathrm{I}}$, in this case, itinerant spins can travel in the same lattice configuration for many times during the averaging.

In order to choose a right value of $N_{1}$, we consider the following temperature dependence of $\tau_{\mathrm{L}}$ in non-frustrated spin systems. The relaxation time is expressed in this case as $[22,23]$ :

$$
\tau_{\mathrm{L}}=\frac{A}{\left|1-T / T_{\mathrm{C}}\right|^{z \nu}},
$$

where $A$ is a constant, $\nu$ - the correlation critical exponent, and $z$ - the dynamic exponent which depend on the spin model and space dimension. For 3D Ising model, $\nu=0.638$ and $z=2.02$. From this expression, we see that as $T$ tends to $T_{\mathrm{C}}, \tau_{\mathrm{L}}$ diverges. In the critical region around $T_{\mathrm{C}}$ the system encounters thus the so-called "critical slowing down": the spin relaxation is extremely long due to the divergence of the spin-spin correlation. When we take into account the temperature dependence of $\tau_{\mathrm{L}}$, the shape of the resistivity is modified strongly at $T_{\mathrm{C}}$ where $\tau_{\mathrm{L}}$ is very long, and in the paramagnetic phase where the relaxation time is very short due to rapid thermal fluctuations. On the other hand, at low $T, \tau_{\mathrm{L}}$ does not modify $\rho$ because in the ordered phase the spin landscape from one microscopic state to another does not change significantly to affect the motion of the itinerant spin (see discussion in Ref. [21]).

\section{Results}

\subsection{Ferromagnets and antiferromagnets}

In ferromagnets, experimental data mentioned above show a peak at $T_{\mathrm{C}}$. The peak is related to the critical slowing-down where the relaxation time diverges. Direct $\mathrm{MC}$ simulations in the case of the Ising spin give a pronounced peak at $T_{\mathrm{C}}$ as shown in Fig. 1 in agreement with experiments. Let us note that $\rho$ increases at low $T$. The reason for this is multiple: it can stem from the freezing or crystallization of itinerant spins at low $T$ or just from the smallness of the number of conduction electrons in such a low- $T$ region. The shape of $\rho$ depends on many factors: lattice structure, various interactions encountered by itinerant spins, electron concentration, relaxation time, spin model, magnetic-field amplitude etc.
For example, a decrease in the interaction between itinerant spins $K_{0}$ will reduce the increase of $\rho$ as $T \rightarrow 0$, an applied magnetic field will decrease the peak height, the larger carrier concentration will reduce $\rho$ in particular at $T_{\mathrm{C}}$. All of these have been discussed in Ref. [19]. We note a strong effect of the temperature dependence of $\tau_{\mathrm{L}}$ on $\rho$ for $T \geq T_{\mathrm{C}}$. This is very important because $\tau_{\mathrm{L}}$ depends intrinsically on the material via $\nu$ and $z$.

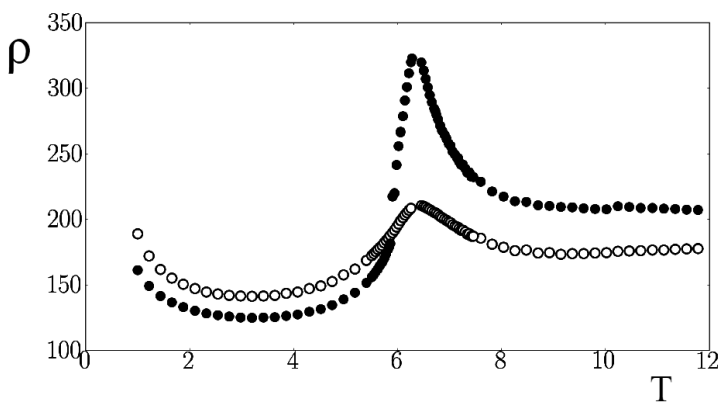

Fig. 1. BCC ferromagnetic and antiferromagnetic films: resistivity $\rho$ with temperature-dependent relaxation for ferro- (black circles) and antiferromagnet (white circles) in arbitrary unit versus temperature $T$, in zero magnetic field, with electric field $\epsilon=1, I_{0}=2$, $K_{0}=0.5, A=1$.

For a quantitative comparison with experiments for a given material, it is necessary to take into account the specific parameters of that material. This is what we do in Sect. 3.3.

In antiferromagnets much less is known because there have been very few theoretical investigations which have been carried out. Haas [24] has shown that while in ferromagnets the resistivity $\rho$ shows a sharp peak at the magnetic transition of the lattice spins, in antiferromagnets there is no such a peak. We found that the peak exists in antiferromagnets but it is less pronounced as seen in Fig. 1. The alternate change of sign of the spinspin correlation with distance may have something to do with the absence of a sharp peak. We have tested for example the effect of the cut-off distance $D_{1}$ [20]: when $D_{1}$ increases, it will include successively up-spin shells and down-spin shells in the sphere of radius $D_{1}$. As a consequence, the difference between the numbers of up and down spins in the sphere oscillates with varying $D_{1}$, making an oscillatory behavior of $\rho$ at small $D_{1}$, unlike in ferromagnets. It is interesting to note that in the presence of an itinerant spin, the ferromagnet and its antiferromagnet counterpart are no more invariant by the local Mattis transformation $\left(J_{i j} \rightarrow-J_{i j}, \boldsymbol{S}_{j} \rightarrow-\boldsymbol{S}_{j}\right)$.

\subsection{Frustrated systems}

We consider the simple cubic lattice shown in Fig. 2. The Hamiltonian is given by

$$
\mathcal{H}=-J_{1} \sum_{(i, j)} \boldsymbol{S}_{i} \cdot \boldsymbol{S}_{j}-J_{2} \sum_{(i, m)} \boldsymbol{S}_{i} \cdot \boldsymbol{S}_{m},
$$

where $\boldsymbol{S}_{i}$ is the Ising spin at the lattice site $i, \sum_{(i, j)}$ is 
made over the NN spin pairs with interaction $J_{1}$, while $\sum_{(i, m)}$ is performed over the NNN pairs with interaction $J_{2}$. We are interested in the frustrated regime. Therefore, hereafter we suppose that $J_{1}=-J(J>0$, antiferromagnetic interaction) and $J_{2}=-\eta J$ where $\eta$ is a positive parameter. The ground state (GS) of this system is easy to obtain either by minimizing the energy, or by comparing the energies of different spin configurations, or just a numerical minimizing by a steepest descent method [25]. We obtain the antiferromagnetic configuration shown by the upper figure of Fig. 3 for $\left|J_{2}\right|<0.25\left|J_{1}\right|$, or the configuration shown in the lower figure for $\left|J_{2}\right|>0.25\left|J_{1}\right|$. Let us note that this latter configuration is 3 -fold degenerate by choosing the parallel NN spins on $x, y$, or $z$ axis. With the permutation of black and white spins, the total degeneracy is thus 6 .

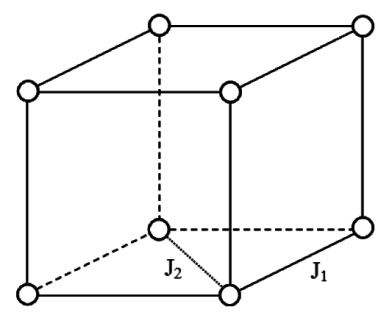

Fig. 2. Simple cubic lattice with nearest and next-nearest neighbor interactions, $J_{1}$ and $J_{2}$, indicated.

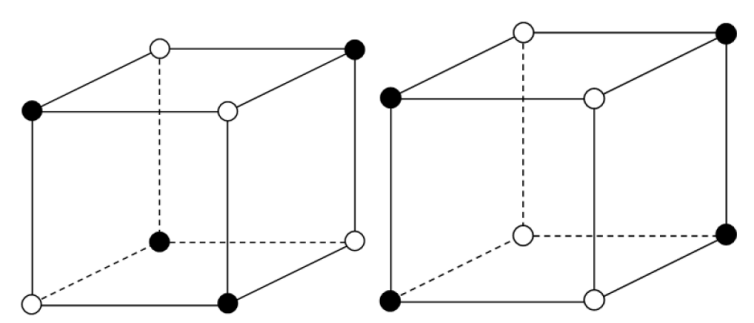

Fig. 3. Simple cubic lattice. Up-spins: white circles, down-spins: black circles. Upper: ground state when $\left|J_{2}\right|<0.25\left|J_{1}\right|$, lower: ground state when $\left|J_{2}\right|>$ $0.25\left|J_{1}\right|$.

The phase transition in the case of the Heisenberg model in the frustrated region $\left(\left|J_{2}\right|>0.25\left|J_{1}\right|\right)$ has been found to be of first order [26]. The system is very unstable due to its large degeneracy. We find that the case of the Ising spin shows an even stronger first-order transition [27]. It is interesting to note that the resistivity of itinerant spins in systems with a first-order transition undergoes a discontinuity at $T_{\mathrm{C}}$ just as the system energy and the order parameter. We show $\rho$ in Fig. 4 for several cut-off distance $D_{1}$. One observes here that $\rho$ can jump or fall at the transition depending on the interaction range $D_{1}$. The resistivity discontinuity has been confirmed in another system with first-order transition, the frustrated fcc antiferromagnet [20]. This seems to be a general rule.

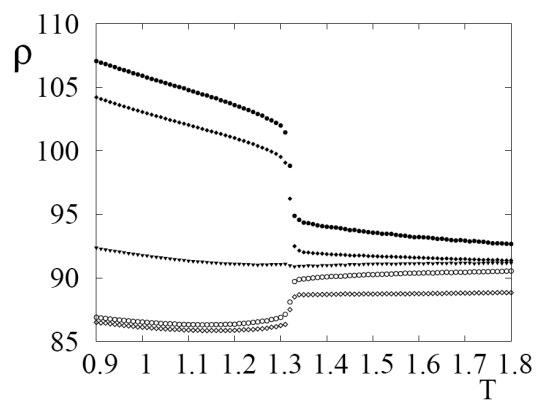

Fig. 4. Spin resistivity versus $T$ for $\left|J_{2}\right|=0.26\left|J_{1}\right|$ for several values of $D_{1}$ : from up to down $D_{1}=0.7 a$, $0.8 a, 0.94 a, a, 1.2 a$. Other parameters are $L_{x}=L_{y}=$ $20, L_{z}=6, I_{0}=K_{0}=0.5, D_{2}=a, D=1, \epsilon=1$.

\subsection{The case of $M n T e$}

The pure MnTe has either the zinc-blende structure [28] or the hexagonal NiAs one shown in Fig. 5. We confine ourselves in the latter case where the Néel temperature is $T_{\mathrm{N}}=310 \mathrm{~K}$ [29]. Hexagonal MnTe is a cross-road semiconductor with a big gap $(1.27 \mathrm{eV})$ and a room-temperature carrier concentration of $n=4.3 \times 10^{17} \mathrm{~cm}^{-3}$ $[30,31]$. Without doping, MnTe is non-degenerate. The behavior of $\rho$ in MnTe as a function of $T$ has been experimentally shown [32-36]. The hexagonal is composed of ferromagnetic $x y$ hexagonal planes antiferromagnetically stacked in the $c$ direction. The $\mathrm{NN}$ distance in the $c$ direction is $c / 2 \approx 3.36 \AA$ shorter than the in-plane NN distance which is $a=4.158 \AA$. Neutron scattering experiments show that the main exchange interactions between Mn spins in MnTe are (i) interaction between NN along the $c$ axis with the value $J_{1} / k_{\mathrm{B}}=-21.5 \pm 0.3 \mathrm{~K}$, (ii) ferromagnetic exchange $J_{2} / k_{\mathrm{B}} \approx 0.67 \pm 0.05 \mathrm{~K}$ between in-plane neighboring Mn (they are next NN by distance), (iii) third $\mathrm{NN}$ antiferromagnetic interaction $J_{3} / k_{\mathrm{B}} \approx-2.87 \pm 0.04 \mathrm{~K}$. The spins are lying in the $x y$ planes perpendicular to the $c$ direction with a small in-plane easy-axis anisotropy $D$ [29]. We note that the values of the exchange integrals given above have been deduced from experimental data by fitting with a formula obtained from a free spin-wave theory [29]. Other fittings with mean-field theories give slightly different values: $J_{1} / k_{\mathrm{B}}=-16.7 \mathrm{~K}, J_{2} / k_{\mathrm{B}}=2.55 \mathrm{~K}$ and $J_{3} / k_{\mathrm{B}}=-0.28 \mathrm{~K}[30]$.

The lattice Hamiltonian is given by

$$
\begin{gathered}
\mathcal{H}=-J_{1} \sum_{(i, j)} \boldsymbol{S}_{i} \cdot \boldsymbol{S}_{j}-J_{2} \sum_{(i, m)} \boldsymbol{S}_{i} \cdot \boldsymbol{S}_{m} \\
-J_{3} \sum_{(i, k)} \boldsymbol{S}_{i} \cdot \boldsymbol{S}_{k}-D_{a} \sum_{i}\left(S_{i}^{x}\right)^{2},
\end{gathered}
$$

where $\boldsymbol{S}_{i}$ is the Heisenberg spin at the lattice site $i, \sum_{(i, j)}$ is made over the NN spin pairs $\boldsymbol{S}_{i}$ and $\boldsymbol{S}_{j}$ with interaction $J_{1}$, while $\sum_{(i, m)}$ and $\sum_{(i, k)}$ are made over the NNN and third NN neighbor pairs with interactions $J_{2}$ and $J_{3}$, respectively. $D_{a}>0$ is an anisotropy constant which 


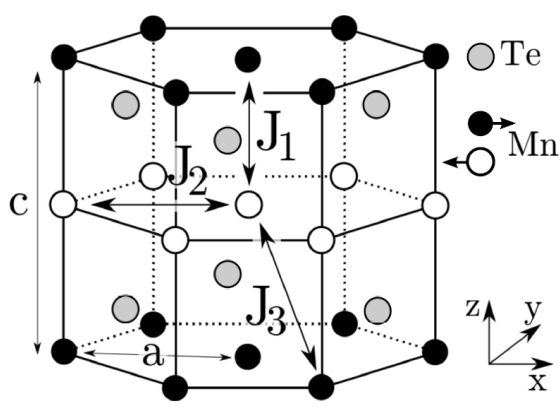

Fig. 5. Structure of MnTe of NiAs type is shown. Antiparallel spins are shown by black and white circles. NN interaction is marked by $J_{1}$, next $\mathrm{NN}$ interaction by $J_{2}$, and third NN one by $J_{3}$.

favors the in-plane $x$ easy-axis spin configuration. The Mn spin is experimentally known to be of the Heisenberg model with magnitude $S=5 / 2$ [29].

The interaction between an itinerant spin and surrounding $\mathrm{Mn}$ spins in semiconducting MnTe is written as

$$
\mathcal{H}_{i}=-\sum_{n} I\left(\boldsymbol{r}-\boldsymbol{R}_{n}\right) \boldsymbol{\sigma} \cdot \boldsymbol{S}_{n},
$$

where $I\left(\boldsymbol{r}-\boldsymbol{R}_{n}\right)>0$ is a ferromagnetic exchange interaction between itinerant spin $\boldsymbol{\sigma}$ at $\boldsymbol{r}$ and Mn spin $\boldsymbol{S}_{n}$ at lattice site $\boldsymbol{R}_{n}$. The sum on lattice spins $\boldsymbol{S}_{n}$ is limited at cut-off distance $D_{1}=a$. We use here the Ising model for the electron spin. In doing so, we neglect the quantum effects which are of course important at very low temperature but not in the transition region at room temperature where we focus our attention. We suppose the following distance dependence of $I\left(\boldsymbol{r}-\boldsymbol{R}_{n}\right)$ :

$$
I\left(\boldsymbol{r}-\boldsymbol{R}_{n}\right)=I_{0} \exp \left(-\alpha\left(\boldsymbol{r}-\boldsymbol{R}_{n}\right)\right),
$$

where $I_{0}$ and $\alpha$ are constants. We choose $\alpha=1$ for convenience. The choice of $I_{0}$ should be made so that the interaction $\mathcal{H}_{i}$ yields an energy much smaller than the lattice energy due to $\mathcal{H}$ (see discussion on the choice of variables given above). Let us note that the cut-off distance is rather short so that the obtained results shown below still keep a general character which does not depend on the choice of exponential form. Since in MnTe the carrier concentration is $n=4.3 \times 10^{17} \mathrm{~cm}^{-3}$, very low with respect to the concentration of its surrounding lattice spins $\approx 10^{22} \mathrm{~cm}^{-3}$, we do not take into account the interaction between itinerant spins.

As mentioned before, the values of the exchange interactions deduced from experimental data depend on the model Hamiltonian, in particular the spin model, as well as the approximations. Furthermore, in semiconductors, the carrier concentration is a function of $T$. In our model, there is however no interaction between itinerant spins. Therefore, the number of itinerant spins used in the simulation is important only for statistical average: the larger the number of itinerant spins the better the statistical average. The current obtained is proportional to the num-

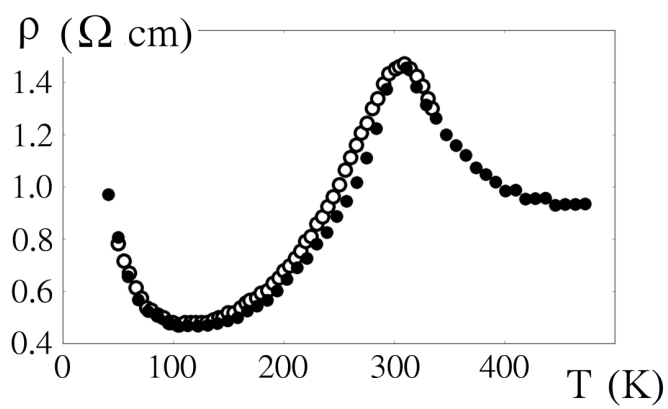

Fig. 6. Spin resistivity $\rho$ versus temperature $T$. Black circles are from Monte Carlo simulation, white circles are experimental data taken from $\mathrm{He}$ et al. [36]. The parameters used in the simulation are $J_{1}=-21.5 \mathrm{~K}$, $J_{2}=2.55 \mathrm{~K}, J_{3}=-9 \mathrm{~K}, I_{0}=2 \mathrm{~K}, D_{a}=0.12 \mathrm{~K}$, $D_{1}=a=4.148 \AA, \epsilon=2 \times 10^{5} \mathrm{~V} / \mathrm{m}, L=30 a$ (lattice size $\left.L^{3}\right)$.

ber of itinerant spins but there are no extra physical effects. Using the exchange integrals slightly modified with respect to the ones given above, we have calculated $\rho$ of the hexagonal MnTe. The result of $\rho$ is shown in Fig. 6. Let us note that with $J_{3}$ slightly larger in magnitude than the value deduced from experiments, we find $T_{\mathrm{N}}=310 \mathrm{~K}$ in excellent agreement with experiments. Furthermore, we observe that $\rho$ shows a pronounced peak and coincides with the experimental data. The values we used to obtain that agreement are $A=1$ and the Heisenberg critical exponents $\nu=0.707, z=1.97$ [23]. In the temperature regions below $T<140 \mathrm{~K}$ and above $T_{\mathrm{N}}$ the $\mathrm{MC}$ result is also in excellent agreement with experiment, unlike in our previous work [18] using the Boltzmann equation.

Using the value of $\rho$, we obtain the relaxation time of itinerant spin equal to $\tau_{\mathrm{I}} \approx 0.1 \mathrm{ps}$, and the mean free path equal to $\bar{l} \approx 20 \AA$, at the critical temperature.

\section{Conclusion}

We have shown in this paper how MC simulations can be used to produce properties of spin transport in magnetic materials. The method is very general, it can be easily applied to a wide range of materials from ferromagnets to antiferromagnets of different lattices and spin models. The results of the spin resistivity $\rho$ as a function of temperature under different situations can be obtained and compared to experiments. We were concentrated in the magnetic phase transition region where theories failed to predict correct behaviors of $\rho$. This is due to the fact that the magnetic resistivity is intimately related to the spin-spin correlation which is very different from one material to another. This correlation, as we know in the domain of phase transition and critical phenomena, governs the nature of the transition: phase transitions of second order of different universality classes and phase transitions of first-order. Needless to say, the nature of the phase transition affects the behavior of $\rho$ as seen above: different shapes of $\rho$ and discontinuity at $T_{\mathrm{C}}$, 
etc. We have, for a good demonstration of the efficiency of our method, studied the case of MnTe where experimental data are recently available for the whole temperature range. Our result is in excellent agreement with experiments: it reproduces the correct Néel temperature as well as the shape of the peak at the phase transition.

\section{References}

[1] J. Xia, W. Siemons, G. Koster, M.R. Beasley, A. Kapitulnik, Phys. Rev. B 79, R140407 (2009).

[2] C.L. Lu, X. Chen, S. Dong, K.F. Wang, H.L. Cai, J.-M. Liu, D. Li, Z.D. Zhang, Phys. Rev. B 79, 245105 (2009).

[3] J. Du, D. Li, Y.B. Li, N.K. Sun, J. Li, Z.D. Zhang, Phys. Rev. B 76, 094401 (2007).

[4] Y.Q. Zhang, Z.D. Zhang, J. Aarts, Phys. Rev. B 79, 224422 (2009).

[5] X.F. Wang, T. Wu, G. Wu, H. Chen, Y.L. Xie, J.J. Ying, Y.J. Yan, R.H. Liu, X.H. Chen, Phys. Rev. Lett. 102, 117005 (2009).

[6] T.S. Santos, S.J. May, J.L. Robertson, A. Bhattacharya, Phys. Rev. B 80, 155114 (2009).

[7] F. Matsukura, H. Ohno, A. Shen, Y. Sugawara, Phys. Rev. B 57, R2037 (1998).

[8] A.E. Petrova, E.D. Bauer, V. Krasnorussky, S.M. Stishov, Phys. Rev. B 74, 092401 (2006).

[9] F.C. Schwerer, L.J. Cuddy, Phys. Rev. 2, 1575 (1970).

[10] T. Kasuya, Prog. Theor. Phys. 16, 58 (1956).

[11] P.-G. de Gennes, J. Friedel, J. Phys. Chem. Solids 4, 71 (1958).

[12] M.E. Fisher, J.S. Langer, Phys. Rev. Lett. 20, 665 (1968).

[13] M. Kataoka, Phys. Rev. B 63, 134435 (2001).

[14] G. Zarand, C.P. Moca, B. Janko, Phys. Rev. Lett. 94, 247202 (2005).

[15] K. Akabli, H.T. Diep, S. Reynal, J. Phys., Condens. Matter 19, 356204 (2007).

[16] K. Akabli, H.T. Diep, J. Appl. Phys. 103, 07F307 (2008).

[17] K. Akabli, H.T. Diep, Phys. Rev. B 77, 165433 (2008).
[18] K. Akabli, Y. Magnin, M. Oko, I. Harada, H.T. Diep, Phys. Rev. B 84, 024428 (2011).

[19] Y. Magnin, K. Akabli, H.T. Diep, I. Harada, Comput. Mater. Sci. 49, S204 (2010).

[20] Y. Magnin, K. Akabli, H.T. Diep, Phys. Rev. B 83, 144406 (2011).

[21] Y. Magnin, Danh-Tai Hoang, H.T. Diep, Mod. Phys. Lett. B 25, 1029 (2011).

[22] P.C. Hohenberg, B.I. Halperin, Rev. Mod. Phys. 49, 435 (1977).

[23] P. Peczak, D.P. Landau, J. Appl. Phys. 67, 5427 (1990).

[24] C. Haas, Phys. Rev. 168, 531 (1968).

[25] V. Thanh Ngo, H.T. Diep, Phys. Rev. B 75, 035412 (2007).

[26] C. Pinettes, H.T. Diep, J. Appl. Phys. 83, 6317 (1998).

[27] Danh-Tai Hoang, Y. Magnin, H.T. Diep, Mod. Phys. Lett. B 25, 937 (2011).

[28] B. Hennion, W. Szuszkiewicz, E. Dynowska, E. Janik, T. Wojtowicz, Phys. Rev. B 66, 224426 (2002).

[29] W. Szuszkiewicz, E. Dynowska, B. Witkowska, B. Hennion, Phys. Rev. B 73, 104403 (2006).

[30] S.R. Mobasser, T.R. Hart, Proc. SPIE, Conf. Series 524, 137 (1985).

[31] J.W. Allen, G. Locovsky, J.C. Mikkelsen, Jr., Solid State Commun. 24, 367 (1977).

[32] S. Chandra, L.K. Malhotra, S. Dhara, A.C. Rastogi, Phys. Rev. B 54, 13694 (1996).

[33] Y.B. Li, Y.Q. Zhang, N.K. Sun, Q. Zhang, D. Li, J. Li, Z.D. Zhang, Phys. Rev. B 72, 193308 (2005).

[34] S.S. Aplesnin, L.I. Ryabinkina, O.B. Romanova, D.A. Balaev, O.F. Demidenko, K.I. Yanushkevich, N.S. Miroshnichenko, Phys. Solid State 49, 2080 (2007); DOI: 10.1134/S106378340711011X.

[35] J.B.C. Efrem D'Sa, P.A. Bhobe, K.R. Priolkar, A. Das, S.K. Paranjpe, R.B. Prabhu, P.R. Sarode, J. Magn. Magn. Mater. 285, 267 (2005); arXiv:cond-mat/0408124v1 (2004).

[36] X. He, Y.Q. Zhang, Z.D. Zhang, J. Mater. Sci. Technol. 27, 64 (2011). 